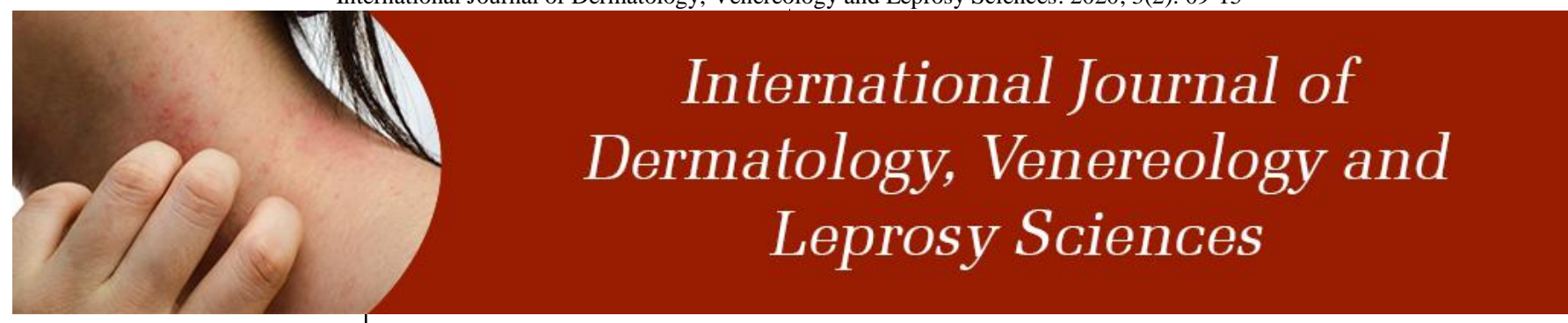

E-ISSN: 2664-942X

P-ISSN: 2664-9411

www.dermatologypaper.com/

Derma 2020; 3(2): 09-15

Received: 07-05-2020

Accepted: 09-06-2020

Dr. Alekhya Ananthula Post Graduate, Department of DVL, Kamineni Institute of Medical Sciences Sreepuram, Narketpally, Nalgonda (District), Telangana, India

Dr. P Vidyasagar Associate Professor, Department of DVL Kamineni Institute of Medical Sciences Sreepuram

Narketpally, Nalgonda (District), Telangana, India

Corresponding Author: Dr. P Vidyasagar

Associate Professor, Department of DVL, Kamineni Institute of Medical Sciences Sreepuram,

Narketpally, Nalgonda (District), Telangana, India

\section{A study on validity of autologous serum skin test in chronic idiopathic urticaria}

\author{
Dr. Alekhya Ananthula and Dr. P Vidyasagar
}

DOI: https://doi.org/10.33545/26649411.2020.v3.i2a.39

\begin{abstract}
Background and Aim: Chronic urticaria is a common distressing dermatosis characterized by spontaneous occurrence of wheals lasting for less than 24 hours, with or without angioedema occurring daily or almost daily for more than 6 weeks. The main aim of the present study is to investigate the positivity of ASST and clinical and severity in chronic idiopathic urticaria.

Methodology: A total number of 100 cases of chronic idiopathic urticaria reporting to the Dermatology Venereology Leprology Department, Kamineni institute of medical sciences were studied.

Results: 46 of them were females and 54 were males. Maximum cases were in the age group of 21-30 years (33\%), followed by age group 31- 40 years (26\%). The age of the youngest patient was 14 years and that of the oldest was 65 years. Autoantibodies, detected by ASST, were seen in the sera of $43 \%$ patients with CIU, comparable to available reports in literature. Presence of autoantibodies was unrelated to gender and the mean age of onset of the disease was earlier in ASST positive patients. Presence of these autoantibodies was significantly associated with more duration of disease, more duration of wheal, frequent attacks of disease and UAS > 5. Angioedema and Abnormal Thyroid profile were significant in ASST positive patients. Dermographism occurred more frequently in ASST Negative patients. There was difference in presentation of clinical symptoms between ASST positive and negative patients but these were not statistically significant. There was difference in CBP, AEC, ESR, RBS Sr IgE Levels and ANA between positive and negative ASST patients, but these results were statistically insignificant.

Conclusion: Thus, ASST identifies a sub group of patients with CIU with more severe disease, who are likely to be more symptomatic and would require more aggressive treatment.
\end{abstract}

Keywords: Utricaria, ASST, Angiodema, Thyroid, Autoantibodies

\section{Introduction}

Urticaria is a common condition - with lifetime incidence of approximately $15 \%$, with females being affected more than males ${ }^{[1]}$. Urticaria is a heterogenous group of diseases defined in common as presence of short lived, blanchable erythematous, edematous, cutaneous swellings or wheals secondary to transient dermal edema and vasodilatation [2] The name "Urticaria" is derived from the Latin word URTICA - meaning to burn or hives ${ }^{[3]}$. Mast cells play a central role in the pathophysiology Pruritus, the primary symptom is associated with significant morbidity ${ }^{[4]}$. Approximately $40 \%$ of patients with urticaria also experience angioedema (swelling that occurs beneath the skin) ${ }^{[5]}$.

Urticaria is generally classified as acute or chronic, depending on the duration of symptoms and the presence or absence of inducing stimuli ${ }^{[6]}$. Chronic urticaria is a common distressing dermatosis characterized by spontaneous occurrence of wheals lasting for less than 24 hours, with or without angioedema occurring daily or almost daily for more than 6 weeks [7, 8]. Chronic idiopathic urticaria, which is synonymous with chronic spontaneous urticaria, is a sub-type of chronic urticarial ${ }^{[9]}$.

In Chronic autoimmune urticaria, circulating immunoglobulin $\mathrm{G}(\mathrm{IgG})$ autoantibodies react to the alpha subunit of the high-affinity IgE receptor on dermal mast cells and basophils, leading to chronic stimulation of these cells and the release of histamine and other inflammatory mediators which cause urticaria and angioedema ${ }^{[10]}$.

The Autologous Serum Skin Test (ASST) has been widely adopted internationally as a clinical test to demonstrate circulating endogenous proinflammatory or wheal-inducing 
factors in urticaria patients since it was first described in $1986^{[11]}$. It is regarded as a reliable in vivo test for chronic idiopathic urticaria with diagnostic, therapeutic and prognostic implications. A positive autologous serum skin test (ASST) is considered to reflect the presence of antiFceRI and/or anti-IgE autoantibodies that are capable of activating mast and basophil cell degranulation ${ }^{[12]}$. Therefore, ASST can be used as a predictive clinical test to diagnose autoimmune urticaria ${ }^{[13,14]}$.

Chronic urticaria is often associated with significant morbidity and a diminished quality of life ${ }^{[15]}$. It is important to determine whether chronic idiopathic urticaria is autoimmune in origin or not. This is especially important since immunosuppressive therapies may be tried if conventional approaches of management are unsuccessful [16]. The present study is undertaken to study positivity of autologous serum skin test in patients suffering with chronic idiopathic urticarial.

Hence the present study emphasize the positivity of ASST and clinical and severity in chronic idiopathic urticaria.

\section{Materials and Methods}

In the present study 100 clinically diagnosed cases of Chronic Idiopathic Urticaria attending the Department of Dermatology, Venereology \& Leprosy at kamineni institute of medical sciences, Narketpally were taken.

The study period was from October 2016 - September 2018 This is an cross sectional study.

\section{Inclusion criteria}

All cases of chronic idiopathic urticaria that is recurrent urticarial wheals of $>6$ weeks duration were taken.

Both Male and Female patients were included.

Patients between the age of $10-70$ yrs were included.

Patients who are not on treatment were included.

\section{Exclusion}

Patients who are on treatment for urticaria were excluded.

Patients on steroids, immunosuppressive treatment and chemotherapy are excluded.

Patients who fail to discontinue medication prior to the test were excluded.

\section{Following details and parameters are noted prior to the test}

Detailed History

Cutaneous and Systemic examination

Investigations

\section{Details included}

Informed consent was taken from all patients

1. Duration of disease

2. Duration of wheals

3. Frequency of attacks

4. No of wheals per attack

5. Intensity of Pruritus

6. Associated Angioedema Or Dermographism

7. Associated Systemic Symptoms (fever, joint pain, abdominal pain etc.)

8. Provoking physical factors

9. Food and Drug Intolerance

10. Associated Medical Illness

11. Urticarial Activity Score

Laboratory investigations which are done to exclude

\section{Systemic causes were}

Complete Blood Picture (CBP)

Complete Urine Examination (CUE)

Erythrocyte Sedimentation Rate (ESR)

Random Blood Sugar (RBS)

Liver Function Tests (LFT)

Renal Function Tests (RFT)

Absolute Eosinophil Count (AEC)

Stool for Ova and Cysts.

Serum IgE Levels

Thyroid Function Tests (TFT)

\section{Urticarial Activity Score ${ }^{[7]}$}

The Urticarial Activity Score (UAS) consisted of the sum of the wheal number score and the itch severity score

Table 1: Urticarial activity score interpretation

\begin{tabular}{|c|c|c|}
\hline Score & Wheals & Pruritis \\
\hline 1 & None & None \\
\hline 2 & $\begin{array}{c}\text { Moderate }(20-50 \\
\text { Wheals/24hr) }\end{array}$ & $\begin{array}{c}\text { Mroublesome but does not } \\
\text { interfere with sleep }\end{array}$ \\
\hline 3 & $\begin{array}{c}\text { Intense }(>50 \text { Wheals/24 } \\
\text { Hrs Or Large Confluent } \\
\text { Area Of Wheals) }\end{array}$ & $\begin{array}{c}\text { Severe pruritus, which is } \\
\text { sufficient troublesome to interfere } \\
\text { with normal daily activity or } \\
\text { sleep }\end{array}$ \\
\hline
\end{tabular}

One has to add both these scores viz., for both the number of wheals and severity of itch, on a given day.

Autologous serum skin test interpretation ${ }^{[17,18]}$

It is based on wheal size and color

a) Wheal Size

0 NEGATIVE CONTROL

1 WHEAL $1.5 \mathrm{~mm}>$ NEGATIVE CONTROL

2 WHEAL $3-5 \mathrm{~mm}>$ NEGATIVE CONTROL

3 WHEAL 6-10mm > NEGATIVE CONTROL SCORE RANGE 0 TO 3
b) Redness Score
0 SKIN COLORED
1 PINK
2 RED (ERYTHEMA)

\section{Statistical analysis}

Descriptive statistics was used to summarize data for comparison between ASST positive and ASST negative group. Chi Square test was used for categorical variables and non parametric test. Man Whitney was used for other variables as they were not

\section{Asst positivity}

ASST was positive in $43(43 \%)$ patients and ASST was negative in $57(57 \%)$ patients.

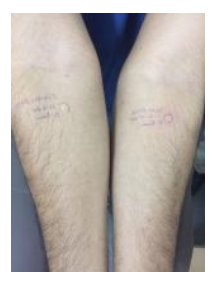

Positive asst 


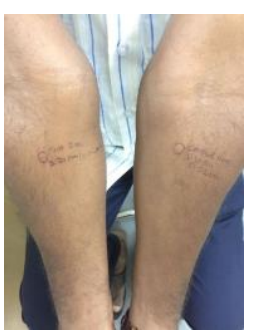

Negative asst

\section{Age wise distribution of patients}

Table 2: Age Wise Distribution

\begin{tabular}{|c|c|c|c|c|c|}
\hline Sl.no & Age & Asst Positive & Asst Negative & Total Patients & Percentage \\
\hline 1 & $10-20$ & 4 & 11 & 15 & $15 \%$ \\
\hline 2 & $21-30$ & 18 & 15 & 33 & $33 \%$ \\
\hline 3 & $31-40$ & 12 & 14 & 26 & $26 \%$ \\
\hline 4 & $41-50$ & 6 & 8 & 14 & $14 \%$ \\
\hline 5 & $51-60$ & 2 & 5 & 7 & $7 \%$ \\
\hline 6 & $61-70$ & 1 & 4 & 5 & $5 \%$ \\
\hline
\end{tabular}

Maximum cases were in the age group of 21-30 years (33\%), followed by age group $31-40$ years $(26 \%)$. The age of the youngest patient was 14 years and that of the oldest was 65 years. The mean age at onset of urticaria in ASST positive patients was $33.06 \pm 11.5$ years while it was $35.24 \pm$ 14.8 years in the ASST negative patients. In the Present Study, age of onset of disease was earlier in positive patients.

Table 3: Gender distribution

\begin{tabular}{|c|c|c|c|c|}
\hline Sl.no & Gender & Total & Asst Positivity & Asst Negativity \\
\hline 1 & FEMALE & 46 & 20 & 26 \\
\hline 2 & MALE & 54 & 23 & 31 \\
\hline
\end{tabular}

Out of 100 patients 46 were females and 54 were males. ASST was positive in $20(46.52 \%)$ female patients and 23 $(53.48 \%)$ male patients. ASST was negative in 26 (45.62\%) female patients and $31(54.38 \%)$ male patients.

Table 4: Duration of the disease

\begin{tabular}{|c|c|c|c|c|}
\hline Sl no & Duration of disease & Asst Positive & Asst Negative & Total \\
\hline 1 & 3 months $-1 \mathrm{yr}$ & 11 & 26 & 37 \\
\hline 2 & $1 \mathrm{yr}-3 \mathrm{yrs}$ & 13 & 13 & 26 \\
\hline 3 & $3 \mathrm{yr}-5 \mathrm{yrs}$ & 12 & 10 & 22 \\
\hline 4 & $>5 \mathrm{yrs}$ & 7 & 8 & 15 \\
\hline
\end{tabular}

The mean duration of the disease in ASST positive patients was $3.35 \pm 2.71 \mathrm{yrs}$ and $2.54 \pm 2.58 \mathrm{yrs}$ in ASST negative patients with $\mathrm{P}$ value $=0.0214$ which was found to be statistically significant. In the present study the duration of disease in ASST positive patients was more as compared to that of ASST negative patients.

Table 5: Duration of the wheals

\begin{tabular}{|c|c|c|c|c|}
\hline Sl no & Duration of wheal & Asst positive & Asst negative & Total \\
\hline 1 & 30 secs - 2hrs & $14(36.6 \%)$ & $30(52.7 \%)$ & 44 \\
\hline 2 & $2-5 \mathrm{hrs}$ & $11(25.6 \%)$ & $9(15.8 \%)$ & 20 \\
\hline 3 & $5-8 \mathrm{hrs}$ & $6(14 \%)$ & $6(10.6 \%)$ & 12 \\
\hline 4 & $8-10 \mathrm{hrs}$ & $10(24 \%)$ & $10(17.6 \%)$ & 20 \\
\hline 5 & $>10 \mathrm{hrs}$ & $2(4.7 \%)$ & $2(3.6 \%)$ & 4 \\
\hline
\end{tabular}

The mean duration of the wheal in ASST positive patients was $4.5 \pm 3.38 \mathrm{hrs}$ and in ASST negative patients was 3.42 \pm 3.22 hrs with $\mathrm{P}$ value $=0.024$ which was found to be statistically significant. In the present study the duration of wheal in ASST positive patients was long lasting as compared to ASST negative patients.

Table 6: Frequency of Attacks

\begin{tabular}{|c|c|c|c|c|}
\hline Sl no & Frequency of attack & Asst positive & Asst negative & Total \\
\hline 1 & Daily & $26(60 \%)$ & $20(35 \%)$ & 46 \\
\hline 2 & $1-4$ episodes/week & $11(26 \%)$ & $23(40 \%)$ & 24 \\
\hline 3 & $1-4$ episodes/month & $6(14 \%)$ & $14(25 \%)$ & 30 \\
\hline
\end{tabular}

In the present study the daily attacks of urticaria was more common in ASST positive patients when compared to ASST negative patients. The percentage of patients with ASST positive were $60 \%$ where as the percentage of ASST negative were $35 \%$ showing a significant $\mathrm{P}$ value $=0.0411$. The percentage of patients having 1-3 attacks per week were $26 \%$ in ASST positive and $40 \%$ in ASST negative patients. The percentage of patients showing in 1-3 urticarial attacks per month were $14 \%$ in ASST positive and $25 \%$ in ASST negative patients

Table 7: Angioedema

\begin{tabular}{|c|c|c|c|c|}
\hline Sl no & Angioedema & Asst positive & Asst negative & Total \\
\hline 1 & Present & $8(18.6 \%)$ & $3(5.27 \%)$ & 11 \\
\hline 2 & Absent & $35(81 \%)$ & $54(94.7 \%)$ & 89 \\
\hline
\end{tabular}

Angioedema occurred in $18.6 \%$ of ASST positive patients and $5.26 \%$ in ASST negative patients with statistically significant $\mathrm{P}$ value of 0.022 .

Table 8: Dermographism

\begin{tabular}{|c|c|c|c|c|}
\hline SI no & Dermographism & Asst positive & Asst negative & Total \\
\hline 1 & Present & $5(11.6 \%)$ & $8(14 \%)$ & 13 \\
\hline 2 & Absent & $38(88.4 \%)$ & $49(86 \%)$ & 87 \\
\hline
\end{tabular}

Dermographism was positive in $11.6 \%$ of ASST Positive patients and $14 \%$ of ASST Negative patients. But these results were statistically insignificant $(\mathrm{P}$ Value $=0.3709)$

Table 9: Clinical Features

\begin{tabular}{|c|c|c|c|}
\hline SI no & Clinical features & Asst positive & Asst negative \\
\hline 1 & Fever & $5(11.6 \%)$ & $1(1.8 \%)$ \\
\hline 2 & Headache & $1(2.3 \%)$ & $12(21 \%)$ \\
\hline 3 & Shortness of breath & $4(9.3 \%)$ & $10(17.6 \%)$ \\
\hline 4 & Atopy & $7(16.3 \%)$ & $2(3.5 \%)$ \\
\hline 5 & Joint pains & $7(16.3 \%)$ & $4(7 \%)$ \\
\hline 6 & Abdominal pain & $5(11.6 \%)$ & $1(1.8 \%)$ \\
\hline 7 & Absent & $14(32.6 \%)$ & $27(47.3 \%)$ \\
\hline
\end{tabular}

ASST positive patients presented with fever, atopy and joint pains more frequently than ASST negative patients, whereas breathlessness and headache was frequently seen in ASST negative patients, however both clinical features were statistically insignificant.

Table 10: Medical illness

\begin{tabular}{|c|c|c|c|}
\hline SI No & Medical illness & Asst positive & Asst negative \\
\hline 1 & Hypertension & $2(4.6 \%)$ & $3(5.3 \%)$ \\
\hline 2 & Diabetes Mellitus & $5(11.6 \%)$ & $10(17.54 \%)$ \\
\hline 3 & Hypothyroidism & $8(18.6 \%)$ & $3(5.3 \%)$ \\
\hline 4 & Absent & $28(65.2 \%)$ & $41(71.86 \%)$ \\
\hline
\end{tabular}

Hypertension was present in $4.6 \%$ of ASST Positive patients 
and $5.3 \%$ of ASST Negative patients. Diabetes Mellitus was present in $11.6 \%$ of ASST Positive patients and $17.54 \%$ of ASST Negative patients. Hypothyroidism was present in $18.6 \%$ of ASST Positive patients and $5.3 \%$ of ASST Negative patients.

Table 11: Uas Score

\begin{tabular}{|c|c|c|c|}
\hline SI no & Uas score & Asst positive & Asst negative \\
\hline 1 & 3 & $1(2 \%)$ & $8(14 \%)$ \\
\hline 2 & 4 & $8(19 \%)$ & $14(25 \%)$ \\
\hline 3 & 5 & $21(49 \%)$ & $29(50 \%)$ \\
\hline 4 & 6 & $13(30 \%)$ & $6(11 \%)$ \\
\hline
\end{tabular}

Urticarial Activity Score > 5 was more common in the ASST positive patients as compared to ASST negative patients, which is statistically significant with $\mathrm{P}$ Value of 0.0272

Table 12: Complete blood picture

\begin{tabular}{|c|c|c|c|}
\hline Sl no & Cbp & Asst positive (\%) & Asst negative (\%) \\
\hline 1 & Anemia & $12(28 \%)$ & $12(21 \%)$ \\
\hline 2 & Leucocytosis & $8(18.60 \%)$ & $9(15.40 \%)$ \\
\hline 3 & Normal & $23(53.40 \%)$ & $36(63.20 \%)$ \\
\hline
\end{tabular}

Complete Blood Picture showed Anemia in 28\% of ASST Positive patients and $21 \%$ of ASST Negative patients. Leucocytosis was positive in $18.6 \%$ of ASST Positive patients and $15.8 \%$ of ASST Negative patients. It was normal in $53.4 \%$ of ASST Positive patients and $63.2 \%$ of ASST Negative patients.

Table 13: Erythrocyte sedimentation rate

\begin{tabular}{|c|c|c|c|}
\hline 0 & ESR & Asst positive (\%) & Asst negative (\%) \\
\hline 1 & RAISED & $3(7 \%)$ & $6(10.50 \%)$ \\
\hline 2 & NORMAL & $40(93 \%)$ & $51(89.50 \%)$ \\
\hline
\end{tabular}

Erythrocyte Sedimentation Rate was positive in $7 \%$ of ASST Positive patients and $10.5 \%$ of ASST Negative patients. It was negative in $93 \%$ of ASST Positive patients and $89.5 \%$ of ASST Negative patients. The values are not statistically significant $(\mathrm{P}$ Value $=0.285)$

Table 14: Random blood sugar

\begin{tabular}{|c|c|c|c|}
\hline Sl no & Rbs & Asst positive (\%) & Asst negative (\%) \\
\hline 1 & Abnormal & $5(11.60 \%)$ & $10(17.50 \%)$ \\
\hline 2 & Normal & $38(89.40 \%)$ & $47(82.50 \%)$ \\
\hline
\end{tabular}

Random Blood Sugar was positive in $11.6 \%$ of ASST Positive patients and $17.5 \%$ of ASST Negative patients. It was negative in $89.4 \%$ of ASST Positive patients and $82.5 \%$ of ASST Negative patients. Values are statistically insignificant. $(\mathrm{P}$ Value $=0.2164)$

Table 15: Absolute eosinophil count

\begin{tabular}{|c|c|c|c|}
\hline SI No & Aec & Asst Positive (\%) & Asst Negative (\%) \\
\hline 1 & Raised & $4(9 \%)$ & $7(12.30 \%)$ \\
\hline 2 & Normal & $39(91 \%)$ & $50(87.70 \%)$ \\
\hline
\end{tabular}

Absolute Eosinophil Count was positive in $9 \%$ of ASST Positive patients and $12.3 \%$ of ASST Negative patients. It was negative in $91 \%$ of ASST Positive patients and $87.7 \%$ of ASST Negative patients with statistically insignificant $\mathrm{P}$
Value $=0.3309$

Table 16: Stool for ova and cysts

\begin{tabular}{|c|c|c|c|}
\hline SI no & Stool for ova and cysts & Asst positive & Asst negative \\
\hline 1 & Present & 0 & 0 \\
\hline 2 & Absent & 43 & 57 \\
\hline
\end{tabular}

Table 17: Serum IgE levels

\begin{tabular}{|c|c|c|c|}
\hline SI no & Serum ige levels & Asst positive & Asst negative \\
\hline 1 & Raised & $2(4 \%)$ & $0(0 \%)$ \\
\hline 2 & Normal & $41(96 \%)$ & $57(100 \%)$ \\
\hline
\end{tabular}

Sr. IgE Level was positive in $4 \%$ of ASST Positive patients and $0 \%$ of ASST Negative patients. It was negative in $96 \%$ of ASST Positive patients and 100\% of ASST Negative patients. The values are statistically insignificant. (P Value $=0.0912$ )

Table 18: Thyroid profile

\begin{tabular}{|c|c|c|c|}
\hline Sl no & $\begin{array}{c}\text { Thyroid } \\
\text { profile }\end{array}$ & $\begin{array}{c}\text { Asst positive } \\
(\%)\end{array}$ & $\begin{array}{c}\text { Asst negative } \\
(\%)\end{array}$ \\
\hline 1 & Abnormal & 0 & 0 \\
\hline 2 & Normal & 43 & 57 \\
\hline
\end{tabular}

\section{Discussion}

Chronic urticaria (CU) is a common skin disorder affecting at least $0.1 \%$ of the population ${ }^{\text {[57] }}$. Mast cell degranulation and histamine release is of central importance in the pathogenesis of $\mathrm{CU}$. About $70 \%$ of the patients with $\mathrm{CU}$ were diagnosed to have chronic idiopathic urticaria (CIU) as no cause could be identified ${ }^{[19]}$.

About $40-50 \%$ of the patients with CIU, demonstrate an immediate wheal and flare response to intra-dermal injected autologous serum. This led to the concept of autoimmune urticaria (AIU). These patients demonstrate circulating histamine-releasing IgG autoantibody against the high affinity IgE receptor FceR1 $\alpha(35-40 \%)$ on dermal mast cells and basophils or less commonly to IgE itself (5 - 10\%) [20].

Basophils and mast cells degranulation leads to release of histamine which is demonstrable by autologous serum skin test (ASST). The ASST; which indicate the presence of functional circulating autoantibodies to FceR $1 \alpha$ and/or to $\operatorname{IgE}$ is the best in vivo clinical test for detection of basophil histamine-releasing activity in vitro. Although basophil histamine-release assay is the gold standard for detecting functional autoantibodies in patient with CIU, this bioassay is difficult to standardize because it requires fresh basophils from healthy donors and is time consuming. ${ }^{14}$ Western blot, enzyme linked immunosorbent assays (ELISA) and flow cytometry may be useful for screening in the future, but they need to be validated.

The present study has evaluated patients with chronic idiopathic urticaria by autologous serum skin testing and compared the clinical features and laboratory parameters of patients with positive and negative ASST results.

\section{Percentage}

In the Present study ASST was positive in $43(43 \%)$ patients and ASST was negative in 57 (57\%) patients.

In study by Vohra et al. ${ }^{[13]}$ ASST was positive in $46(46 \%)$ and negative in the other $54(54 \%)$ patients. 


\section{Age distribution of the patients}

In the Present study, CIU was found to be more common among the age groups of 21-30 years (33\%), followed by $31-40$ years $(26 \%)$. Mean age of onset was $33.06 \pm 11.5 \mathrm{yrs}$ in ASST Positive patients and 35.24 $\pm 14.8 \mathrm{yrs}$ in ASST Negative patients indicating earlier age of onset in Positive patients.

Zeinab Abdel Azim et al. ${ }^{[21]}$ found that the mean age in ASST positive group was $34.3 \pm .10 .2$ years, while that of ASST negative group was $29.6 \pm 11.1$ years

\section{Gender distribution}

In the Present study, Out of 100 patients 46 were females and 54 were males. ASST was positive in 20 (46.52\%) female patients and $23(53.48 \%)$ male patients. ASST was negative in $26(45.62 \%)$ female patients and 31 (53.38\%) male patients.

Mamatha et al. ${ }^{[22]}$ study observed that of a total of 100 patients, 56 were females and 44 males. 18 out of the 34 ASST-positive patients $(52.94 \%)$ and 38 out of the 66 ASST-negative patients $(57.58 \%)$ were female, but there was no statistical difference between males and females.

According to A. Ganapathi et al. ${ }^{[23]}$, females had a higher percentage $(46.4 \%)$ of ASST positivity than males (35\%), but this was not statistically significant $(\mathrm{P}=0.43)$.

\section{Duration of disease}

In the Present study mean duration of the disease in ASST positive patients mean+ S.D is $3.35 \pm 2.71$ yrs and $2.54 \pm$ $2.58 \mathrm{yrs}$ in ASST negative ( $\mathrm{P}$ value $=0.0214)$, which is significant. Duration of Disease was more in positive patients than in negative patients.

A significant increase $(\mathrm{P}$ value $=0.002)$ in the duration of the disease in ASST positive group (median 4 years) in comparison to that of ASST negative group (median 1 year) was observed by Zeinab Abdel Azim et al. ${ }^{[21]}$.

All the above studies are in correlation with the present study.

\section{Duration of Wheal}

The mean duration of the wheal in ASST positive patients is $4.5 \pm 3.38 \mathrm{hrs}$ and in ASST Negative patients it is $3.42 \pm$ 3.27 hrs with $\mathrm{P}$ value of 0.024 , which is statistically significant, indicating duration of wheal is longer in Positive patients.

Mamatha et al. ${ }^{[22]}$ observed that Wheals lasted for significantly longer duration in patients with positive ASST. The median duration being $4 \mathrm{hrs}$ for ASST-positive patients compared to $2 \mathrm{hrs}$ in ASST-negative individuals $(\mathrm{P}=$ $0.001)$, which was statistically significant. These results were in correlation with the present study

\section{Frequency of attacks}

The percentages of patients with ASST positive were $60 \%$ whereas the percentage with ASST negative were 35\% showing a significant $P$ value (0.0411). 1-3 attacks per week $26 \%$ were ASST positive and $40 \%$ were ASST negative. The percentage of patients showing in 1-3 urticarial attacks per month were 14\% ASST positive and 25\% ASST negative. Patients with ASST positivity were seen to have daily attacks of urticaria when compared to ASST negative patients, indicating that frequent urticarial attacks are more in ASST Positive patients than that of ASST Negative patients
Zeinab Abdel Azim et al. [21] study shows that the percentage of patients with more frequent attacks (> 5/week) were significantly higher in positive ASST group $(93.3 \%)$ compared to ASST negative $(65 \%)$ group ( $\mathrm{p}=$ 0.048). This was statistically significant.

\section{Angioedema}

According to the current study angioedema is present in 18.6\% ASST positive patients and 5.26\% in ASST negative patients with a statistically significant $P$ value $=0.02$

In Nettis et al. [25] study, the prevalence (69\%) of angioedema is significantly higher in the ASST-positive cases as compared with that $(43 \%)$ in the ASST-negative patients.

\section{Dermographism}

In the present study dermographism was present in the $11.6 \%$ of ASST positive patients and $14 \%$ of negative patients, which was statistically not significant $(\mathrm{P}$ Value $=$ 0.3709)

Zeinab Abdel Azim et al. ${ }^{[21]}$ study observed that $80 \%$ of ASST positive patients show dermographism, while $60 \%$ in the ASST negative group, This does not show any statistical significance.

\section{Clinical features}

According to Present Study, ASST Positive patients presented with fever, atopy and joint pains more frequently than ASST negative patients, whereas shortness of breath and headache were frequently seen in ASST negative patients, however both clinical features were statistically insignificant.

Vohra et al. ${ }^{[26]}$ study shows that Multiple symptoms were associated in 24(52\%) ASST-positive and 20 (37\%) ASST negative patients respectively. The gastrointestinal symptoms like abdominal pain, diarrhoea, indigestion (13 patients), general malaise, headache, loss of concentration, lassitude, feverish feel and feeling of hot or cold (45 patients), breathlessness/wheezing, palpitations (12 patients) and joint pains (two patients) were more frequent in the ASST-positive cases while symptoms like nausea/vomiting (three patients), flushing (seven patients), joint swelling (one patient) and syncope (three patients) were seen more often in those with negative ASST. However, the difference was not statistically significant $(P=0.18)$.

Urticarial activity score

In the Present study Urticarial Activity Score is $>5$ was more common in ASST positive patients as compared to ASST negative patients, which is statistically significant.

$(\mathrm{P}$ Value $=0.0272)$

In Zeinab Abdel Azim et al. ${ }^{[21]}$ there was significant increase $(\mathrm{P}$ Value $=0.005)$ in UAS in ASST positive group (UAS mean \pm SD $4.5 \pm 0.5$ ) in comparison to ASST negative group (UAS mean \pm SD $3.9 \pm 0.7$ ).

\section{Complete blood picture}

In the Present Study, Anemia was present in $28 \%$ of ASST positive patients and $21 \%$ of ASST negative patients. Leucocytosis is present in $18.6 \%$ of the ASST positive patients and $15.8 \%$ of ASST negative patients. But these values were not statistically significant where $P$ value was 0.611

Krupa shanker et al. ${ }^{[27]}$ study found that leucocytosis was present in the $12.1 \%$ of the ASST negative patients and 
none of them with ASST positive. They also found that the level of haemoglobin was low in $3.1 \%$ of ASST negative patients and $6.4 \%$ in ASST positive patients. But this was not statistically significant. This is in correlation with the present study.

\section{Erythryocyte sedimentation rate}

In the Present Study, Erythrocyte Sedimentation Rate was $7 \%$ in ASST Positive patients and $10.5 \%$ in ASST Negative patients which was higher in ASST Negative patients.

\section{Absolute eosinophilic count}

In the Present Study AEC was raised in 9\% of ASST positive patients and $12.3 \%$ of ASST negative patients. This was not statistically significant $(\mathrm{P}$ Value $=0.3309)$

Krupa shanker et al. ${ }^{[27]}$ study observed that raised AEC was seen in $12.1 \%$ of ASST negative patients and $8.5 \%$ of ASST positive patients, which is not statistically significant.

\section{Thyroid profile}

In the Present Study, Thyroid abnormality was present in $18.26 \%$ of ASST positive patients and $5.26 \%$ of ASST negative patients. This was statistically significant with $\mathrm{P}$ Value $=0.0221$

Mamatha et al. ${ }^{[22]}$ observed abnormal TFT values in 3 ASST-positive patients. This study did not find any difference in the incidence of thyroid disease. The above results were not in correlation with the present study. This might be due to low incidence of thyroid abnormalities in ASST positive patients in that study.

\section{Conclusion}

ASST is a simple, out- patient procedure, inexpensive, semi invasive and easy-to-perform test It can be used as a simple screening test to diagnose chronic idiopathic urticaria patients. It is a cost-effective test for detecting Chronic Autoimmune Urticaria patients who have no distinctive clinical features differentiating them from Chronic Idiopathic Urticaria patients. It is especially important from the therapeutic point of view as it may help the dermatologist to initiate immunosuppressive therapy in such patients.

\section{Acknowledgement}

The author is thankful to Department of DVL for providing all the facilities to carry out this work.

\section{Conflict of Interest}

None

\section{References}

1. An approach to the patient with urticaria S J Deacock clin Exp Immunol. 2008; 153(2):151-161.

2. Clive EH, Ruth A, Malcom W. Chronic urticaria. J Am Acad Dermatol. 2002; 46:645-57.

3. Sachdeva S, Gupta V, Amin SS, Tahseen M. Chronic urticaria. Indian J Dermatol. 2011; 56:622-8.

4. Maurer M, Bindslev-Jensen C, Gimenez-Arnau A, Godse K, Grattan CEM, Hide $\mathrm{M}$ et al Chronic idiopathic urticaria is no longer idiopathic: time for an update! Br J Dermatol. 2013; 168:455-456.

5. Amar SM, Dreskin SC. Urticaria. Prim Care 2008; 35:141157.

6. Urticaria angioedema: Amin Kanani, Robert
Schellenberg and Richard Warrington; Allergy, Asthma \& Clinical Immunology, 2011.

7. Godse KV. Urticaria meter. Indian J Dermatol 2012; 57:410-1.

8. Tseng JTP, Lee WR, Lin SS, Hsu CH, Yang HH, Wang $\mathrm{KH}$ et al. Autologous serum skin test and autologous whole blood injections to patients with chronic urticaria: A retrospective analysis. Dermatol Sinica. 2009; 27:27-36.

9. Sanchez Borges M, Asero R, Ansotegui IJ, Baiardini I, Bernstein JA, Canonica GW, et al. WAO Scientific and Clinical Issues Council: Diagnosis and treatment of urticaria and angioedema: a worldwide perspective. WAO J. 2012; 5:12547.

10. Poonawalla T, Kelly B. Urticaria: a review. 2009; 10:921.

11. Grattan CE, Wallington TB, Warin RP, Kennedy CT, Bradfield JW. A serological mediator in chronic idiopathic urticaria-a clinical, immunological and histological evaluation. Br J Dermatol. 1986; 114:583590.

12. Guttman-Yassky, Bergman R, Maor C, Mamorsky M, Pollack S, Shahar E. The autologous serum skin test in a cohort of chronic idiopathic urticaria patients compared to respiratory allergy patients and healthy individuals. J Eur Acad Dermatol Venereol. 2007; 21(1):35-9.

13. Vohra S, Sharma NL, Mahajan VK. Autologous serum skin test: Methodology, interpretation and clinical applications. Indian J Dermatol Venereol Leprol. 2009; 75:545-8.

14. Godse KV. Autologous serum skin test in chronic idiopathic urticaria. Indian J Dermatol Venereol Leprol. 2004; 70:283-4.

15. Weldon DR. Quality of life in patients with urticaria. Allergy Asthma Proc. 2006; 27:96-99.

16. Grattan CE, Sabroe RA, Greaves MW. Chronic urticaria. J Am Acad Dermatol. 2002; 46:645-57.

17. Toubi E, Kessel E, Avshovich N, Bamberger, Sabo E, Nusem D, et al. Clinical and laboratory parameters in predicting chronic urticaria duration: A prospective study of 139 patients. Allergy. 2004; 59:869-73.

18. Grattan CEH. Autoimmune urticaria. Immunol Allergy Clin N Am. 2004; 24:163-81.

19. Kulthanan K, Jiamton S, Gorvanich T, Pinkaew S. Autologous serum skin test in chronic idiopathic urticaria: Prevalence, correlation and clinical implications. Asian Pac J Allergy Immunol. 2006; 24:201-6.

20. Soundararajan S, Kikuchi Y, Joseph K, Kaplan AP. Functional assessment of pathogenic IgG subclasses in chronic autoimmune urticaria. J Allergy Clin Immunol. 2005; 115:815-21.

21. Autologous Serum Skin Test in Chronic Idiopathic Urticaria: Comparative Study in Patients with Positive versus Negative Test Zeinab Abdel Azim M.D., Shaymaa El Mongy M.D. and Hanan Salem M.D. J Egypt Women Dermatol Soc. 2010; 7:129-133.

22. George M, Balachandran C, Prabhu S. Chronic idiopathic urticaria: Comparison of clinical features with positive autologous serum skin test. Indian $\mathbf{J}$ Dermatol Venereol Leprol. 2008; 74:105-8.

23. Adaikalampillai Ganapathy Vikramkumar, Sheela Kuruvila, Satyaki Ganguly. Autologous serum skin test 
as an indicator of chronic autoimmune urticaria in a tertiary care hospital in South India, Department of Dermatology, Venereology and Leprosy, Pondicherry Institute of Medical Sciences, Puducherry, India, Indian Dermatol Online J. 2014; 5(S2):87-91.

24. Staubach P, Onnen K, Vonend A, Metz M, Siebenhaar $\mathrm{F}$, Tschentscher I et al. Autologous whole blood injections to patients with chronic urticaria and a positive autologous serum skin test: a placebocontrolled trial. Dermatology. 2006; 212:150-159.

25. Nettis E, Dambra P, D Oronzio L, Cavallo E, Loria MP, Fanelli $\mathrm{M}$ et al. Reactivity to autologous serum skin test and clinical features in chronic idiopathic urticaria. Clin Exp Dermatol. 2002; 27:29-31.

26. Surbhi Vohra N and Lal Sharma, Vikram K Mahajan, Vinay Shanker. Clinicoepidemiologic features of chronic urticaria in patients having positive versus negative autologous serum skin test: A study of 100 Indian patients IJDVL. 2011; 77(2):156-159.

27. Krupashankar DS, Shashikala K, Rama Madala. Clinical and Investigative Assessment of Patients with Positive versus Negative Autologous Serum Skin Test; A Study of 80 South Indian Patients. Indian J Dermotol. 2012; 57:434-8. 